\title{
Neural activity associated with episodic memory for emotional context
}

\author{
E.J. Maratos ${ }^{a}$, R.J. Dolan ${ }^{\text {b,c }}$, J.S. Morris ${ }^{b}$, R.N.A. Henson ${ }^{\mathrm{a}, \mathrm{b}}$, M.D. Rugg ${ }^{\mathrm{a}, *}$ \\ a Institute of Cognitive Neuroscience and Department of Psychology, 17, Queen Square, University College London, London WC1N 3AR, UK \\ ${ }^{\mathrm{b}}$ Wellcome Department of Cognitive Neurology, 12, Queen Square, Institute of Neurology, London WC1N 3BG, UK \\ ${ }^{\mathrm{c}}$ Royal Free Hospital School of Medicine, Roland Hill Street, London NW3 $2 P F, U K$
}

Received 17 November 2000; received in revised form 31 January 2001; accepted 2 February 2001

\begin{abstract}
To address the question of which brain regions subserve retrieval of emotionally-valenced memories, we used event-related fMRI to index neural activity during the incidental retrieval of emotional and non-emotional contextual information. At study, emotionally neutral words were presented in the context of sentences that were either negatively, neutrally or positively valenced. At test, fMRI data were obtained while participants discriminated between studied and unstudied words. Recognition of words presented in emotionally negative relative to emotionally neutral contexts was associated with enhanced activity in right dorsolateral prefrontal cortex, left amygdala and hippocampus, right lingual gyrus and posterior cingulate cortex. Recognition of words from positive relative to neutral contexts was associated with increased activity in bilateral prefrontal and orbitofrontal cortices, and left anterior temporal lobe. These findings suggest that neural activity mediating episodic retrieval of contextual information and its subsequent processing is modulated by emotion in at least two ways. First, there is enhancement of activity in networks supporting episodic retrieval of neutral information. Second, regions known to be activated when emotional information is encountered in the environment are also active when emotional information is retrieved from memory. (C) 2001 Elsevier Science Ltd. All rights reserved.
\end{abstract}

Keywords: Emotion; Episodic memory; fMRI; Negative; Positive; Neuroimaging

\section{Introduction}

A small number of functional neuroimaging studies have investigated the brain regions and circuits activated during explicit retrieval of emotionally toned items. In one of the first such studies it was reported that activity in occipital-parietal regions was enhanced during visualisation from memory of previously viewed negative images relative to neutral images [27]. The finding of enhanced activity in sensory regions during emotional memory retrieval was replicated by Taylor et al. [58], who found greater activity in the lingual gyrus (BA 18) during recognition of previously studied negative images, relative to neutral images, in the absence of the modulation of any other retrieval-related activity.

\footnotetext{
* Corresponding author. Tel.: + 44-20-76791120; fax: +44-2078132835 .

E-mail address: m.rugg@ucl.ac.uk (M.D. Rugg).
}

In a more recent study, Dolan et al. [11] reported that recognition memory for emotional relative to neutral pictures was associated with activation in the anterior temporal pole and the amygdala, leading these authors to conclude that emotional memory retrieval recruited brain regions specialised for processing the affective significance of stimuli.

The present investigation was concerned with characterising the brain activity associated with retrieval of emotionally toned contexts rather than retrieval of items with inherent emotional tone. To the best of our knowledge, only one earlier neuroimaging study [33] has investigated the neural correlates of retrieval of such contexts. The results of this electrophysiological study suggested that activity in the same network that supports episodic retrieval of neutral information is enhanced during retrieval of emotional information. Specifically, it was suggested that the incidental retrieval of emotional contextual information was associ- 
ated with enhanced activity in the same neural systems that support the conscious recollection and subsequent 'post-retrieval' processing of emotionally neutral information. In particular, it was suggested that such additional activity would be found in the medial temporal/posterior cortical regions subserving the initial retrieval of episodic information [37,49] and in right prefrontal regions supporting the subsequent monitoring and evaluation of the retrieved information (e.g. [48]).

The present study employed event-related fMRI to investigate the incidental retrieval of emotional and neutral contexts using a procedure similar to that employed by Phelps et al. [39] (see also [33]) which, unlike most prior studies of emotional memory, did not confound the emotional valence of the retrieval cues with the valence of the to-be-retrieved information. By contrasting the neural activity elicited by a single kind of test item (emotionally neutral words) as a function of the emotional valence of its study context, any difference in the activity elicited by test items belonging to different classes of context can be attributed unequivocally to their respective encoding contexts.

\section{Method}

The experimental procedures were approved by the joint Medical Ethics Committee of the National Hospital for Neurology and Neurosurgery and the Institute of Neurology.

\subsection{Participants}

Thirteen right-handed participants aged between 18 and 30 years were employed in the study after giving informed consent. One participant's data were rejected because of very poor behavioural performance. The 12 participants whose data were analysed consisted of seven females and five males. All reported good health and no history of neurological illness.

\subsection{Experimental material}

One hundred and twenty words normed for neutral emotional valence (see below) were used as critical stimuli. The words varied in length between four and ten letters and in frequency between 30 and 100 per million [14]. For each word one negative, one neutral, and one positive sentence were constructed. For example, for the critical word 'corn' the following sentences were generated as negative, neutral and positive sentences, respectively. 'The farmer was shredded when he fell into the corn grinder'; 'The farm labourers began harvesting the corn'; and 'The farmer was overjoyed with his bountiful crop of corn'. Critical words never featured in sentences other than their designated neutral, negative or positive sentences.

\subsection{Word norms and selection}

Normative valence ratings were collected for 320 ostensibly neutral words. The words were randomly intermixed with 35 emotionally negative words and 35 emotionally positive words (taken from Siegle [53]). Ten participants were given the resulting list of 390 words and asked to rate each word on a Likert scale (ranging from -3 to +3 ) according to how emotionally 'negative', 'neutral' or 'positive' they felt it to be. The average ratings given to the negative, neutral and positive words were $-1.8,0.4$ and 1.6 , respectively. Of the 320 'neutral' words, 21 were dropped because they received ratings outside the range \pm 1 , another 131 were dropped because of difficulties in constructing a negative, neutral or positive sentence, and 48 words were used as filler items. The remaining 120 words were used as critical stimuli.

\subsection{Experimental lists}

Eight study lists were created, each containing a set of 15 negative, 15 neutral and 15 positive sentences. The sentences in the study lists were randomly ordered and a neutral filler sentence was added to the beginning and end of each study list. Six test lists were created, each with a different randomised order, such that each study list could be followed by a test list containing the 45 old words from the sentences in the study block and 15 previously unseen words. Two filler words were added to the beginning of each test list.

To accommodate the two study-test sessions, study lists were paired such that across the whole experiment there were four study sets (each set containing a list for session 1 and corresponding list for session 2). Test lists were similarly paired, resulting in three test sets. Study and test sets were then combined resulting in 12 possible study-test set combinations, one for each participant.

Study and test lists were assigned to participants such that, across the experiment, each critical word appeared three times as a new item and three times each in a negative, neutral and positive sentence.

\subsection{Procedure}

Participants were given a description of the study task before entering the scanner. They were informed that the experiment would involve the presentation of emotional sentences and that parts of the experiment would involve a memory task. An example of the study phase was then given which consisted of the presentation of 3 negative, 3 neutral and 3 positive sentences. 
The experiment proper began after a 15 min structural scan. Participants were then administered the study and test lists in the scanner. An interval of 5 min separated each of the study and test tasks and also each of the sessions. Functional scans were acquired during each of the two test phases.

\subsection{Task}

Each of the 45 study trials consisted of two stages. A series of 45 sentences, white text on a dark screen, were presented via a mirror mounted on the scanner head coil in direct view of the reclining participant at a viewing distance of approximately $50 \mathrm{~cm}$. First, the participants were required to give the sentence an affective rating on a Likert scale from -3 to +3 according to how negative, neutral or positive they felt the sentences to be. The critical word from the sentence was then presented alone with the requirement to read it out aloud and remember it in the context of the sentence of which it had been part. Sentences were presented approximately every $10-15 \mathrm{~s}$.

The corresponding recognition memory test consisted of the re-presentation of the critical words from the study sentences along with 15 new items. Each trial began with the presentation of a fixation character for $1000 \mathrm{~ms}$. This was removed $500 \mathrm{~ms}$ before the onset of the test word which was displayed for $1000 \mathrm{~ms}$, following which the screen was blanked for $3000 \mathrm{~ms}$. Participants were required to judge whether or not each word had been presented in the study phase. No mention was made of the different types of sentences employed at study. Test stimuli were presented in a white uppercase Helvetica 48 font on a black background. The words were presented using the same display system as for the study phase. Responses were given through a hand-held box by button press with either the right or left thumb. The mapping of thumb to response type was counterbalanced across participants. Participants were instructed to relax and to keep head movement to a minimum. Responses faster than $300 \mathrm{~ms}$ were treated as errors.

\section{7. fMRI methods}

A $2 \mathrm{~T}$ Siemens VISION system (Siemens, Erlangen, Germany) was used to acquire both T1-weighted anatomical volume images $(1 \times 1 \times 1.5 \mathrm{~mm}$ voxels, MPRAGE sequence) and T2*-weighted echoplanar (EPI) images $(64 \times 64,3 \times 3 \mathrm{~mm}$ pixels, $\mathrm{TE}=40 \mathrm{~ms})$ with blood oxygenation level dependent (BOLD) contrast. Each EPI consisted of 32 2-mm thick axial slices (inter-slice gap $1.5 \mathrm{~mm}$ ), positioned to cover the entire brain with the exception of the vertex and cerebellum. Scanning occurred during the two test sessions. Thus, 109 volumes were acquired in each session, the first 5 of which were discarded to allow for $\mathrm{T} 1$ equilibration effects. Volumes were acquired continuously with an effective repetition time (TR) of $3.2 \mathrm{~s} / \mathrm{vol}$. The SOA was $5.5 \mathrm{~s}$, giving an effective sampling rate of $10 \mathrm{~Hz}$.

\subsection{Image pre-processing}

Pre-processing steps were conducted using the Statistical Parametric Mapping toolbox [2]. For each participant, all volumes were realigned and re-sampled in space using sinc-interpolation. Temporal offsets between successive slices were corrected with sinc-interpolation in time. The data were spatially normalised to a standard EPI template volume (based on the MNI 305 brain average) using non-linear basis functions [2]. Finally, the EPI volumes were convolved with an isotropic $8 \mathrm{~mm}$ (FWHM) Gaussian smoothing kernel to accommodate residual across-participant anatomical differences, and globally scaled to a constant mean signal intensity. The time series for each voxel were high-pass filtered to a maximum of $1 / 120 \mathrm{~Hz}$.

\subsection{Data analysis}

Statistical maps of voxels exhibiting differential responses to the different event types were created with SPM 99b [16]. The volumes acquired during each session were treated as two time series. The analysis consisted of two stages, estimation of the parameters of the haemodynamic response function for each participant and a subsequent test of the significance of these parameters across participants.

In the first stage, responses to the onset of each event type were modelled with two basis functions; an early haemodynamic response function ('canonical' HRF: [17]) and a delayed HRF, shifted in time relative to the early function by $3.2 \mathrm{~s}$ (i.e., one TR). The use of both an early and late basis function was based on findings (e.g. [51]) that the haemodynamic response onsets later in some brain regions (e.g. prefrontal cortex) than it does in the sensory regions from which the canonical (early) HRF was derived.

The early and late response functions, when convolved with a sequence of delta functions representing the onset of each event, were used as participant-specific covariates in a general linear model. Since the two HRFs were correlated, covariates for the late HRF were orthogonalised with respect to those for the early HRF [1]. This step was taken to ensure that no variance was shared between the two response functions. The parameter estimates for a series of planned contrasts were obtained in each participant's data separately for the early and late covariates. All the fMRI contrasts were confined to test trials associated with correct decisions. 
The participant-specific contrast images were entered into one sample $t$-tests $(\mathrm{df}=11)$. Regions sensitive to recognition memory regardless of study context were identified as those voxels common to each of the three 'old-new' contrasts (neutral-new; negative-new; positive-new; each thresholded at $P<0.05$ uncorrected). For the planned contrasts between the old words belonging to each type of context a statistical threshold of $t=4.02$ was employed, corresponding to an uncorrected $P<0.001$. Only activations involving contiguous clusters of at least 4 voxels were interpreted. The maxima of suprathreshold regions were localised using a combination of the participants' normalised structural images and the stereotaxic atlas of Talairach and Tournoux [57].

\section{Results}

\subsection{Behavioural data}

The mean valence for the negative, neutral and positive sentences were 2.38 (S.D. $=0.52), 0.21(0.14)$ and $1.61(0.28)$, respectively. These means differed reliably $[(F(1.20,12.80)=327.82, P<0.001) ;$ d.f.s adjusted by the Greenhouse-Geisser procedure]. A $t$-test contrasting the absolute differences between negative and neutral ratings (mean $=2.60$ ) and positive and neutral ratings $($ mean $=1.40)$ was also significant $(t(11)=$ 64.93, $P<0.001)$, indicating that the negative contexts attracted the more extreme ratings.

Hit rates for words from the negative, neutral and positive sentences, along with correct rejection rates and associated reaction times, are shown in Table 1. ANOVA conducted on the proportion of correct responses (negative hits, neutral hits, positive hits, correct rejections) revealed a main effect of condition $(F(2.7$, $29.50)=12.59, P<0.001)$. Tukey HSD tests revealed that the participants made a higher proportion of correct responses to new words than to any type of old word. Planned pairwise comparisons between the classes of old items (negative, neutral, positive) were not significant, nor were analogous comparisons between reaction times for these items.

Table 1

Behavioural data from experiment ${ }^{\mathrm{a}}$

\begin{tabular}{cllll}
\hline & CR & Negative hit & Neutral hit & Positive hit \\
\hline Proportion & 0.85 & 0.68 & 0.63 & 0.69 \\
& $(0.14)$ & $(0.14)$ & $(0.15)$ & $(0.15)$ \\
Reaction & 1283 & 1184 & 1198 & 1177 \\
time (ms) & $(330)$ & $(361)$ & $(330)$ & $(296)$ \\
\hline
\end{tabular}

\footnotetext{
a Mean proportions (S.D. in brackets) of correct rejections, negative hits, neutral hits and positive hits along with associated reaction times.
}

\section{2. fMRI data}

\subsubsection{Old versus new}

As illustrated in Fig. 1 and documented in Table 2, a number of regions were identified that were common to the three possible old versus new contrasts. Among these regions were left lateral parietal cortex, precuneus, anterior and posterior cingulate, and bilateral prefrontal cortex. These regions correspond closely to those reported in several other recent event-related fMRI studies of recognition memory (e.g. [21,26]; see [47] for review).

\subsection{Retrieval of negative context}

Regions sensitive specifically to the retrieval of negative context were identified by contrasts between the responses elicited by old items from negative vs. neutral contexts (Table 3). The contrast employing the early HRF revealed several regions with enhanced responses to negative items (Table 3a). These regions included right dorsolateral prefrontal cortex, cuneus, and lateral and medial temporal regions including bilateral superior temporal cortex, left anterior temporal cortex, hippocampus, amygdala and primary auditory cortex. The same contrast modelled with the late HRF (Table $3 b)$ revealed activation in temporal lobe regions including left superior temporal cortex and left insula, as well as activation in right posterior cingulate, parahippocampal, and extrastriate cortex.

A subset of the foregoing regions were also identified in the contrast between responses elicited by items from the negative vs. positive contexts. When modelled with the early HRF enhanced activity was found in the cuneus (44 voxels, $x y z=-4,-84,20, Z=4.84$ ) and anterior temporal cortex (6 voxels $x y z=-48,6,4$, $Z=3.23$ ). Enhanced activity was also found in the left anterior insula when the threshold was reduced to $P<0.005$ (81 voxels $x y z=-40,2,6, Z=2.80)$. When responses were modelled with the late HRF enhanced activity was found in the left anterior insula (12 voxels, $x y z=-44,10,-4, Z=3.67)$. Enhanced activity was also found in superior temporal lobe when the threshold was reduced to $P<0.005$ (5 voxels, $x y z=-$ $56,-50,12, Z=2.87)$.

\subsection{Retrieval of positive context}

Regions showing a greater response to items from the positive versus neutral contexts are given in Table 4 . These regions included right anterior prefrontal cortex, left inferior prefrontal cortex, bilateral orbitofrontal cortex and an anterior medial temporal region (Table 4a). Two further regions, right precentral gyrus and left inferior prefrontal cortex, were identified from the contrast performed on parameter estimates derived from the late covariate (Table 4b). 


\section{Early}
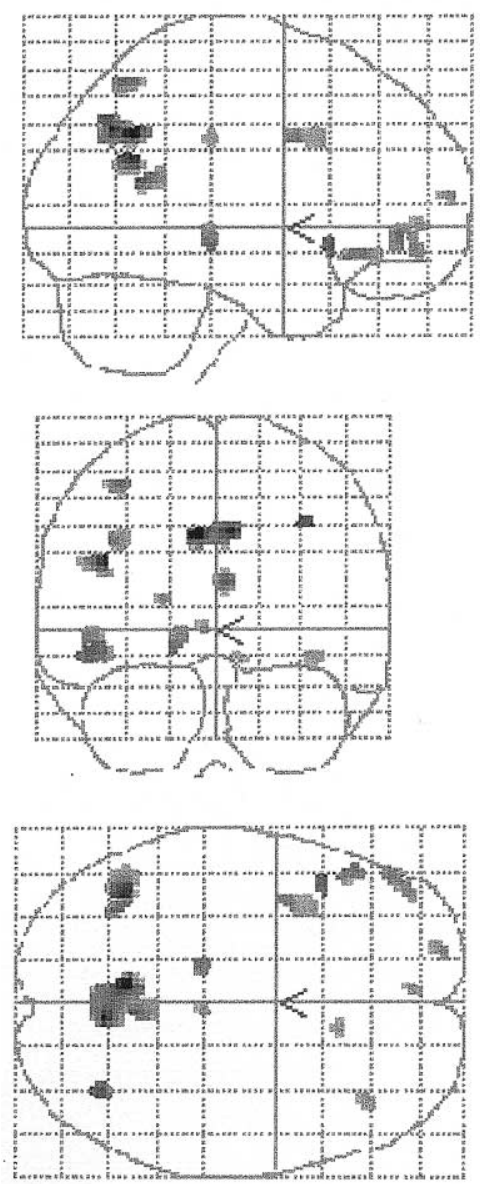

Late
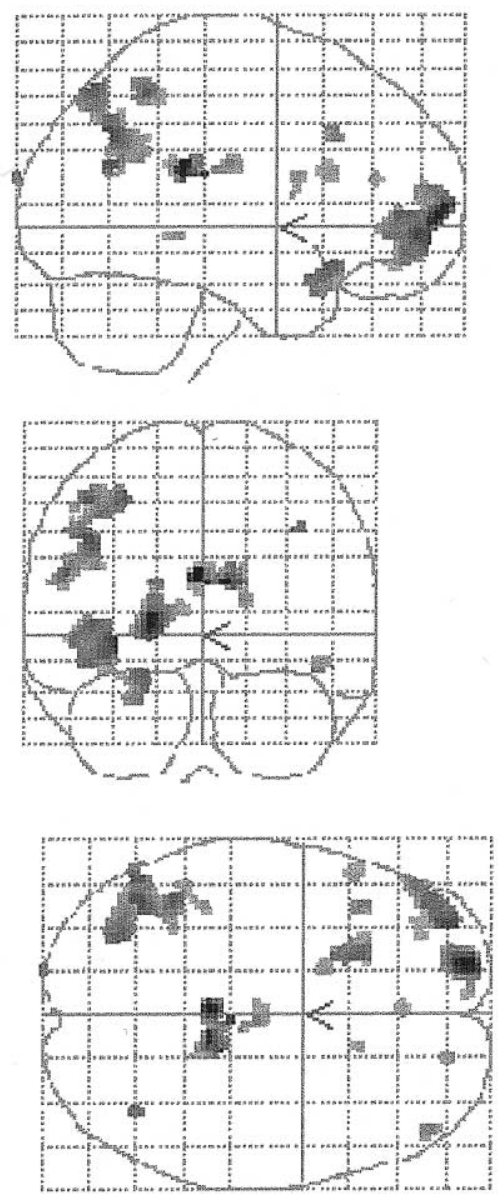

Fig. 1. Maximum intensity projections illustrating voxels common to each of the three 'old-new' contrasts (see text); Early: regions identified by modelling responses with the early HRF. Late: regions identified using the late HRF (see Section 2).

The contrast between the positive and negative contexts revealed enhanced activity for items from the positive contexts in only one of the foregoing areas, namely the left orbitofrontal cortex (14 voxels $x y z=-$ $2,46,-18, Z=3.52$ ). However, the same effect was also observed in the right orbitofrontal cortex when the threshold was reduced to $P<0.005$ (63 voxels $x y z=2$, $36,-24, Z=3.04)$.

Selected regions activated during the retrieval of negative and positive contexts are shown in Fig. 2 and Fig. 3 respectively. Percent signal change associated with contrasts between responses to items from neutral contexts with those from each of the two emotional contexts are shown for selected regions in Fig. 4.

\subsection{Valence-nonspecific activations}

Voxels common to the contrasts employed to investigate valence-specific effects were assessed by masking the results of one of the contrasts by the other, both thresholded at $P<.001$. No regions of overlap were found.

\section{Discussion}

\subsection{Behavioural performance}

Items encoded in the neutral, negative and positive contexts were recognised with equivalent levels of accuracy, and with comparable RTs. These findings are inconsistent with previous reports that emotionally valenced contexts enhance memory (as indexed by free recall) for pre-experimentally neutral stimuli $[39,40]$. However, there is evidence that the effects of emotionality on memory increase with time [28], so it is possible that the effects of the present emotionality manipulation would have become more evident with a longer study-test interval. It is also possible that simple yes/no recognition memory, as opposed to recall, was not sufficiently sensitive to detect differences in memory arising from the context manipulation. Indeed, in a study with an encoding task similar to the one employed here [33], items studied in emotional contexts were more likely to be recognized than were items from 
neutral contexts, and were also more likely to be attributed to the correct 'source' (i.e. emotional vs. neutral sentence). These findings suggest that the context manipulation employed here most likely did modulate episodic memory. On a positive note, the failure to find context effects on recognition accuracy or RT in the present study makes it unlikely that differences in the BOLD responses elicited by items from the different contexts reflect differences attributable to variables such as speed or confidence of associated recognition judgements.

The finding that negative sentences received more extreme valence ratings than did the positive sentences complicates the interpretation of the fMRI findings. Whereas effects that were larger for the retrieval of positive than negative contexts can be attributed with some confidence to valence-sensitive processes, this is not true for effects in the opposite direction. In this case, it is not possible to distinguish between the possibilities that the fMRI effects reflect processes sensitive specifically to the retrieval of negative memories, as opposed to the retrieval of memories eliciting relatively high levels of emotional 'arousal' [19].

\section{2. $f M R I$ data}

The principal aim of this study was to identify neural systems activated by incidental retrieval of contextual emotional information by using a novel procedure which unconfounds the emotionality of the retrieval cue and to-be-retrieved information. The findings indicated that retrieval of such information was associated with activation of several regions previously implicated in emotional processing (see [30] for review), including the amygdala and orbitofrontal cortex. In addition, retrieval of items encoded in emotional, relative to neu-

Table 2

Maxima within regions showing common signal increases (to 3 old/new contrasts, see text) on the early (a) and late (b) covariates for the old versus new contrast

\begin{tabular}{lllll}
\hline Region of activation & $\mathrm{L} / \mathrm{R}$ & Number of voxels & & Talairach co-ordinates \\
\cline { 3 - 5 } & & $x$ & $y$
\end{tabular}

\section{(a) Early}

Superior frontal

Medial frontal

Medial frontal

Middle frontal

Inferior frontal

Inferior frontal

Inferior frontal

Precentral

Parahippocampal

Cingulate

Posterior Cingulate

Precuneus

Superior temporal/inferior parietal

Superior parietal

Inferior parietal

(b) Late

Superior frontal

Superior frontal

Middle frontal

Middle frontal

Middle frontal

Anterior cingulate

Anterior cingulate

Inferior frontal

Inferior frontal

Inferior frontal

Posterior cingulate

Posterior cingulate

Middle temporal

Inferior parietal

Inferior parietal

Inferior parietal

Cuneus

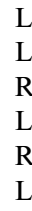

-20
-4
10
-48
38
-52
-48
-38
-16
4
4
-6
-42
-36
34

64
52
24
44
34
30
18
4
-28
-28
-54
-58
-58
-62
-68

$\begin{array}{rr}12 & 10 \\ 0 & 10 \\ -12 & 11 \\ -4 & 10 \\ -10 & 47 \\ -10 & 47 \\ -8 & 47 \\ 34 & 6 \\ -8 & 28 \\ 34 & 31 \\ 18 & 23 \\ 36 & 7 \\ 26 & 39 \\ 56 & 7 \\ 40 & 7\end{array}$

$\begin{array}{rrrr}-20 & 64 & 6 & 10 \\ 16 & 56 & 14 & 10 \\ -34 & 54 & -4 & 10 \\ 46 & 48 & -14 & 11 \\ -42 & 22 & 36 & 8 \\ -2 & 38 & 20 & 32 \\ 12 & 22 & 22 & 32 \\ -22 & 22 & -16 & 47 \\ -52 & 20 & 20 & 45 \\ -16 & 8 & 20 & 44 \\ 2 & -18 & 24 & 23 \\ -2 & -36 & 24 & 23 \\ -42 & -38 & -4 & 21 \\ -44 & -50 & 52 & 40 \\ -44 & -64 & 38 & 39 \\ 36 & -64 & 40 & 40 \\ -16 & -100 & 20 & 18\end{array}$


Table 3

Maxima within regions showing significant $(P<0.001$ uncorrected) signal increases for early (a) and late (b) covariates for the negative versus neutral contrast

\begin{tabular}{|c|c|c|c|c|}
\hline \multirow[t]{2}{*}{ Region of activation } & \multirow[t]{2}{*}{ Number of voxels } & Talairach co-ordinates & \multirow[t]{2}{*}{ Peak $Z$} & \multirow[t]{2}{*}{ Brodmann area } \\
\hline & & $y$ & & \\
\hline
\end{tabular}

\section{(a) Early}

Middle/inferior frontal

Inferior frontal

Anterior temporal

Amygdala

Anterior temporal

Hippocampus

Transverse temporal

Middle temporal

Superior temporal

Superior temporal

Cuneus/precuneus

$\begin{array}{lr}\text { R } & 34 \\ \text { R } & 4 \\ \text { L } & 17 \\ \text { L } & 4 \\ \text { R } & 5 \\ \text { L } & 19 \\ \text { L } & 28 \\ \text { R } & 10 \\ \text { R } & 12 \\ \text { L } & 17 \\ \text { L } & 15\end{array}$

44
54
-30
-26
-40
-30
-44
66
64
-62
-8

30
24
6
-8
-14
-18
-22
-28
-42
-34
-78

$\begin{array}{rll}12 & 3.88 & 46 \\ 2 & 3.68 & 45 \\ -18 & 3.81 & 34 \\ -24 & 3.46 & 34 \\ -16 & 3.36 & 28 / 34 \\ -20 & 3.89 & 28 \\ 12 & 3.89 & 41 \\ 0 & 3.84 & 21 \\ 18 & 3.43 & 22 \\ 18 & 3.42 & 22 \\ 24 & 3.69 & 18\end{array}$

(b) Late

Superior frontal

Middle frontal

Precentral

Insula

Parahippocampal

Superior temporal

Posterior cingulate

Lingual

$\begin{array}{lr}\mathrm{L} & 17 \\ \mathrm{R} & 8 \\ \mathrm{R} & 12 \\ \mathrm{~L} & 9 \\ \mathrm{R} & 8 \\ \mathrm{~L} & 12 \\ \mathrm{R} & 49 \\ \mathrm{R} & 23\end{array}$

-24
34
50
-46
12
-50
4
26

44
42
4
0
-52
-56
-54
-64

30
36
18
-2
-2
16
22
0

$\begin{array}{ll}3.79 & 9 \\ 3.73 & 9 \\ 3.69 & 44 / 6 \\ 3.52 & 22 \\ 3.41 & 30 / 19 \\ 3.5 & 22 / 39 \\ 3.83 & 23 / 31 \\ 3.82 & 18 / 19\end{array}$

Table 4

Maxima within regions showing significant $(P<0.001)$ signal increases on the early (a) and late (b) covariates for the positive versus neutral contrast

\begin{tabular}{|c|c|c|c|c|}
\hline \multirow[t]{2}{*}{ Region of activation } & \multirow[t]{2}{*}{ Number of voxels } & Talairach co-ordinates & \multirow[t]{2}{*}{ Peak $Z$} & \multirow[t]{2}{*}{ Brodmann area } \\
\hline & & $x$ & & \\
\hline
\end{tabular}

\section{(a) Early}

Superior/middle frontal

Medial orbitofrontal

Medial orbitofrontal

Inferior frontal

Inferior frontal

Anterior temporal

Fusiform

Superior temporal

Middle occipital

Inferior occipital

$\begin{array}{lr}\mathrm{R} & 5 \\ \mathrm{R} & 7 \\ \mathrm{~L} & 13 \\ \mathrm{~L} & 9 \\ \mathrm{R} & 4 \\ \mathrm{~L} & 6 \\ \mathrm{~L} & 5 \\ \mathrm{~L} & 4 \\ \mathrm{R} & 7 \\ \mathrm{R} & 4\end{array}$

36
6
-4
-48
60
-36
-54
-44
30
42

$+2$

$$
z
$$

$\begin{array}{rrll}58 & 18 & 3.43 & 10 \\ 48 & -18 & 3.45 & 11 \\ 46 & -14 & 3.51 & 11 \\ 30 & 10 & 3.84 & 46 \\ 8 & 14 & 3.27 & 44 \\ 4 & -18 & 3.83 & 34 \\ -10 & -24 & 3.27 & 20 \\ -38 & 16 & 3.46 & 22 \\ -84 & 16 & 3.59 & 18 \\ -86 & -6 & 3.5 & 18\end{array}$

(b) Late

Precentral

Inferior frontal

$\begin{array}{ll}\mathrm{R} & 8 \\ \mathrm{~L} & 4\end{array}$

50
-28

0
14

$\begin{array}{lrr}16 & 4.89 & 6 \\ 14 & 4.63 & 44\end{array}$

tral contexts was associated with activation in regions thought to play a more general role in episodic retrieval, notably the prefrontal cortex and hippocampus. Since a single class of retrieval cue (neutral words) was employed to elicit both neutral and emotional memories, these findings cannot be attributed to differential retrieval 'sets' that may be adopted when the emotional valence of the retrieval cue predicts the valence of the stored information (the situation in most studies of emotional memory), or to any other kind of interaction between emotionally valenced cues and the memories they elicit.

Relative to items from neutral contexts, items from the negative contexts elicited enhanced activity in left amygdala. This finding does not, however, appear to be strongly valence-specific; the direct contrast between items from negative and positive contexts failed to reveal differential amygdala activity and, as can be seen 


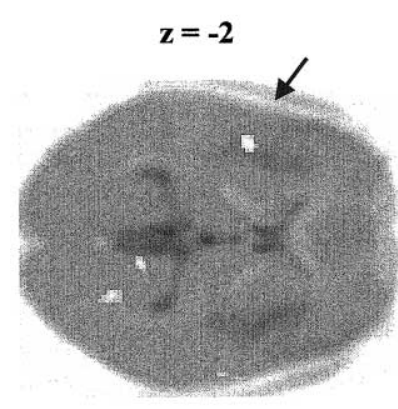

Insula

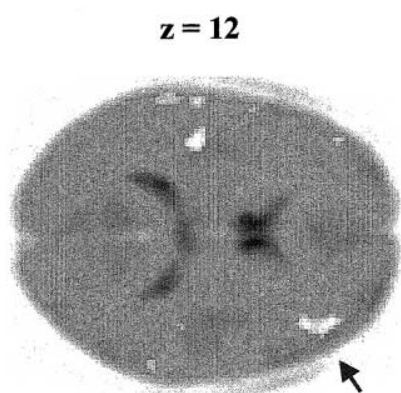

Right Prefrontal

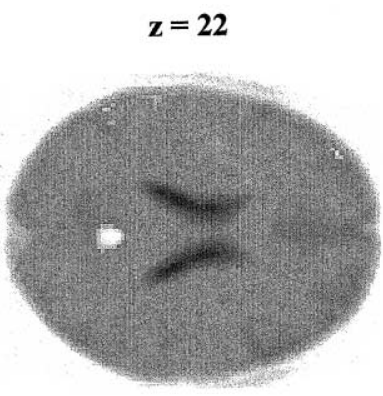

Posterior cingulate

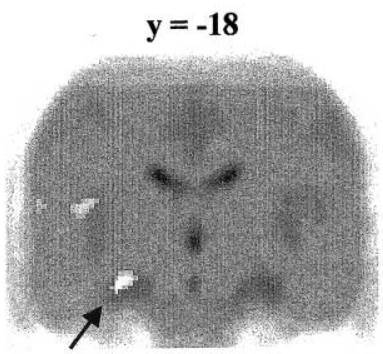

Hippocampus

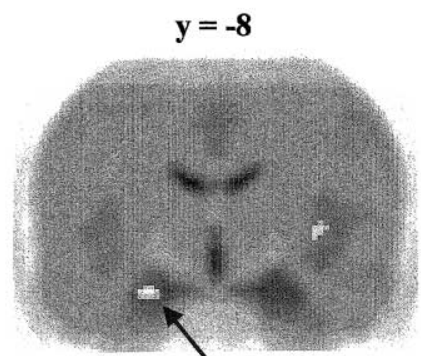

Amygdala

Fig. 2. Selected regions identified by the contrast between items from negative vs. neutral contexts (thresholded at $P<0.005$ uncorrected), displayed on normalised EPI images averaged across all participants. Hippocampal, amygdala and right prefrontal cortex were identified in contrasts employing the early HRF, insula and posterior cingulate were identified with the late HRF (see Section 2).

in Fig. 4, items from the positive contexts also tended to elicit activity in this structure $(z=2.62, P<0.005)$. The finding of amygdala activation during the incidental retrieval of emotional context builds on the recent finding of the engagement of this structure during recognition memory of emotional items, regardless of their valence [11]. These authors proposed that the role of the amygdala in episodic retrieval was to tag retrieved memories with 'representations of their behavioural significance' [59]. The present findings are consistent with this proposal, in as much as it implies that amygdala activation is a consequence, rather than a precursor, of the retrieval of emotionally valenced episodic memories. As noted already, because of the use of a single class of retrieval cue, activity dependent on the encoding context of the cue must have followed, rather than preceded, the retrieval of the contextual information.

The finding of orbitofrontal cortex activation during the retrieval of positive contexts is consistent with evidence that this region plays a role in emotional processing. For example, previous neuroimaging studies have reported orbitofrontal activation during the recollection of happy and sad life events $[18,38]$, and in the appraisal of reward [44]. In addition, clinical studies have shown that orbitofrontal lesions are associated with abnormal social behaviour $[10,46]$ hypothesised to result from impairments in decision making ([3]; also see [9]), and in the control and correction of rewardand punishment-related behaviour [45]. The present
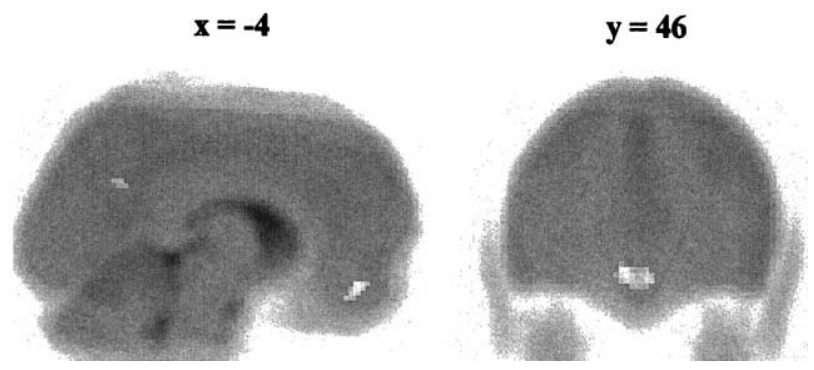

Fig. 3. Orbitofrontal regions identified by the contrast between items from positive vs. neutral contexts using the early HRF (thresholded at $P<0.005$ uncorrected). Note that the activated regions are in tissue unaffected by susceptibility artefact.

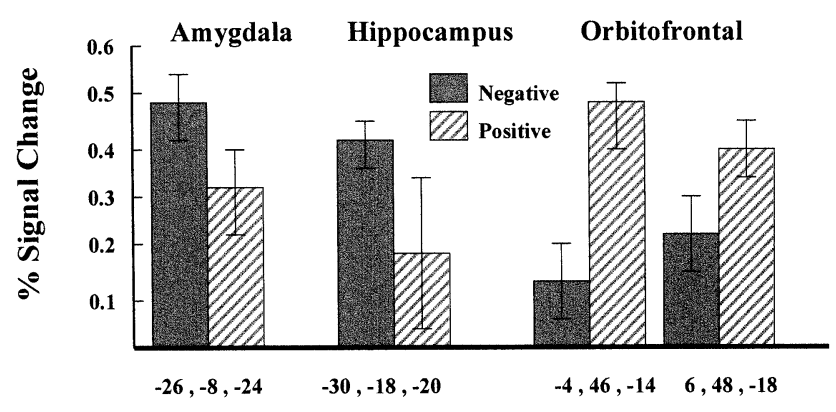

Fig. 4. Percent signal change and associated standard errors for positive vs. neutral and negative vs. neutral contrasts at regions indicated. Contrasts used the early HRF (see Section 2). 
findings add further to the foregoing observations in that they suggest that orbitofrontal cortex is engaged by the retrieval of memories that have been associated with positive (and, presumably, 'rewarding') emotional contexts. Furthermore, the sensitivity of this region for retrieval of emotionally positive contextual information suggests that in some circumstances orbitofrontal cortex is engaged preferentially by emotionally positive, rather than emotionally negative, information (c.f. [36]).

Other than the amygdala and orbitofrontal cortex, we identified several other regions which previous research has implicated in the processing of emotion, including anterior temporal cortex [11,25], posterior cingulate cortex $[15,32,41]$, and anterior insula $[6,29,42]$. The finding that retrieval of both negative and positive contextual information is associated with anterior temporal activation accords with Mesulam's [35] proposal that this region plays a role in imparting affective valence to experience. With respect to the findings for the posterior cingulate, it is noteworthy that Maddock [31] proposed that the caudal part of the posterior cingulate cortex [including but not limited to the retrosplenial cortex and encompassing the posterior cingulate' regions (BA 23, 31) activated in the present study] acts as an 'interface' between emotion and episodic memory. The present findings are consistent with this proposal. However, posterior cingulate activation has been reported in a number of recent event-related studies of episodic retrieval of emotionally neutral information $[12,20,21]$. The present findings may, therefore, reflect enhancement of activity in circuits subserving episodic retrieval in general, rather than systems specialised for the processing of emotion.

Finally, left anterior insula, identified in both the negative vs. neutral and negative vs. positive contrasts, has been linked with the processing of disgust [54], generation of sadness [29,43] and emotional learning and fear conditioning [6]. The present findings, which are consistent with these observations, may reflect the fact that autonomic activity triggered by the retrieval of negative contexts was greater than that triggered by retrieval of positive or neutral information. This suggestion is consistent with the proposal that the insula is involved in representing somatic states [8].

In addition to the findings for regions thought to support emotional processing, retrieval of the both positive and negative contexts was associated with the enhanced activity in regions implicated more generally in episodic memory retrieval (for reviews see $[4,7,34,55])$. Most notable among these regions were left and right prefrontal cortex, and left hippocampus. Several proposals have been advanced as to the functional significance of right prefrontal activation during episodic retrieval $[24,48,51,52]$. The present findings are most consistent with the proposal that this region supports 'post-retrieval' processes, specifically, operations involved in the monitoring and evaluation of the products of retrieval [21,22,52]. According to this interpretation, retrieval of emotional contexts, either by virtue of the amount or the salience of the retrieved information, engaged these post-retrieval processes to a greater extent than did retrieval of emotionally neutral information. It is also possible, however, to interpret the right frontal effects observed here in terms of the 'retrieval effort' hypothesis of right prefrontal function in episodic retrieval [50]; by this argument, the effects reflect the additional resources allocated to recover emotional versus non-emotional information.

Another region implicated in episodic memory and identified in the present study was the hippocampus. For reasons that remain unclear, hippocampal activation in episodic retrieval tasks has been observed inconsistently in functional neuroimaging studies ([47]; but see [56]), and this is one of the first event-related studies to report such activation (see also [13]). There are two possible kinds of interpretation for this finding. It may reflect a role for the hippocampus in the recollection of contextually- or information-rich memories. This interpretation is consistent both with the finding that items from negative versus neutral contexts elicit greater 'recollection-related' ERP effects [33], and with the results of previous PET and fMRI studies [13,49]. Alternatively, the greater hippocampal activity for items from negative contexts may reflect processing 'downstream' from contextual retrieval. For example the activity might form part of a re-instatement of the initial encoding episode, or reflect 're-encoding' operations set in train by the retrieval of emotionally arousing or salient information.

The foregoing discussion is predicated on the assumption that the effects we observed reflect the consequences of the explicit retrieval of the test words' study contexts. It is possible, however, that some of these effects reflect memory in the form of conditioned emotional responses, brought about the association of the study words with the emotions aroused by the contexts in which they were embedded. For example, stimuli that have been associated with negative emotion have been shown in several studies to activate the amygdala (see [5] for review; see also [23]). We cannot discount the possibility that similar processes may have been operating in the present study. One aim of future research should be to dissociate the effects of these two forms of memory.

In summary, we provide evidence that neural activity mediating episodic retrieval and subsequent processing of retrieved information are modulated in at least two ways by emotion. First, there is enhancement of activity in regions subserving episodic retrieval in general. Second, regions activated when emotional information is encountered externally in the environment are reactivated when that information is subsequently retrieved. 


\section{Acknowledgements}

E.J. Maratos is supported by the UK Medical Research Council. R.J. Dolan, J.S. Morris, R.N.A. Henson and M.D. Rugg are supported by the Wellcome Trust.

\section{References}

[1] Andrade A, Paradis AL, Rouquette S, Poline JB. Ambiguous results in functional neuroimaging data analysis due to covariate correlation. Neuroimage 1999;10:483-6.

[2] Statistical Parametric Mapping SPM 99b. Wellcome Department of Cognitive Neurology, London, UK, 1999.

[3] Bechara A, Damasio H, Damasio AR. Emotion, decision making and the orbitofrontal cortex. Cerebral Cortex 2000;10:295307.

[4] Buckner RL. Neuroimaging of memory. In: Gazzaniga MS, editor. The New Cognitive Neurosciences. Cambridge, MA: MIT Press, 2000:817-29.

[5] Büchel C, Dolan RJ. Classical fear conditioning in functional neuroimaging. Current Opinion in Neurobiology 2000;10:21923.

[6] Büchel C, Dolan RJ, Armony JL, Friston KJ. Amygdalahippocampal involvement in human aversive trace conditioning revealed through event-related functional magnetic resonance imaging. Journal of Neuroscience 1999;19:10869-76.

[7] Cabeza R, Nyberg L. Imaging cognition II: an empirical review of 275 PET and fMRI studies. Journal of Cognitive Neuroscience 2000;12:1-47

[8] Craig AD, Chen K, Bandy D, Reiman EM. Thermosensory activation of insular cortex. Nature Neuroscience 2000;3:184-90.

[9] Damasio AR. The Feeling of What Happens: Body and Emotion in the Making of Consciousness. New York: Harcourt Brace, 1999.

[10] Damasio AR, Tranel D, Damasio H. Individuals with sociopathic behavior caused by frontal damage fail to respond autonomically to social stimuli. Behavioural Brain Research 1990;41:81-94.

[11] Dolan RJ, Lane R, Chua P, Fletcher P. Dissociable temporal lobe activations during emotional episodic memory retrieval. Neuroimage 2000;11:203-9.

[12] Donaldson DI, Petersen SE, Ollinger JM, Buckner RL. Dissociating item and state components of recognition memory using fMRI. Neuroimage, in press.

[13] Eldridge LL, Knowlton BJ, Furmanski CS, Bookheimer SY, Engel SA. Remembering episodes: a selective role for the hippocampus during retrieval. Nature Neuroscience 2001;3:1149-52.

[14] Francis WN, Kucera H. Frequency Analysis of English Usage: Lexicon and Grammar. Boston: Houghton Mifflin Company, 1982.

[15] Fredrikson M, Wik G, Fischer H, Andersson J. Affective and attentive neural networks in humans: a PET study of Pavlovian conditioning. Neuroreport 1995;7:97-101.

[16] Friston KJ, Holmes AP, Worsley KJ, Poline JB, Frith CD, Frackowiak RSJ. Statistical parametric maps in functional imaging: a general linear approach. Human Brain Mapping 1995;2:189-210.

[17] Friston KJ, Josephs O, Rees G, Turner R. Non-linear event-related responses in fMRI. Magnetic Resonance in Imaging 1998;39:41-52.

[18] George MS, Ketter TA, Parekh PI, Horwitz B, Herscovitch P, Post RM. Brain activity during transient sadness and happiness in healthy women. American Journal of Psychiatry 1995; $152: 341-51$.

[19] Heilman KM, Gilmore RL. Cortical influences in emotion. Journal of Clinical Neurophysiology 1998;15:409-23.

[20] Henson RN, Rugg MD, Shallice T, Dolan RJ. Confidence in recognition memory for words: Dissociating right prefrontal roles in episodic retrieval. Journal of Cognitive Neuroscience, in press.

[21] Henson RN, Rugg MD, Shallice T, Josephs O, Dolan RJ. Recollection and familiarity in recognition memory: an event-related functional magnetic resonance imaging study. Journal of Neuroscience 1999;19:3962-72.

[22] Henson RN, Shallice T, Dolan RJ. Right prefrontal cortex and episodic memory retrieval: a functional MRI test of the monitoring hypothesis. Brain 1999;122:1367-81.

[23] Isenberg N, Silbersweig D, Engelien A, Emmerich S, Malavade $\mathrm{K}$, Beattie B, et al. Linguistic threat activates the human amygdala. Proceedings of the National Academy of Sciences; 96:10456-59.

[24] Kapur S, Craik FI, Jones C, Brown GM, Houle S, Tulving E. Functional role of the prefrontal cortex in retrieval of memories: a PET study. Neuroreport 1995;6:1880-4.

[25] Kimbrell TA, George MS, Parekh PI, Ketter TA, Podell DM, Danielson $\mathrm{AL}$, et al. Regional brain activity during transient self-induced anxiety and anger in healthy adults. Biological Psychiatry 1999;46:454-65.

[26] Konishi S, Wheeler ME, Donaldson DI, Buckner RL. Neural correlates of episodic retrieval success. Neuroimage 2000;12:276-86.

[27] Kosslyn SM, Shin LM, Thompson WL, McNally RJ, Rauch SL, Pitman RK, et al. Neural effects of visualizing and perceiving aversive stimuli: a PET investigation. Neuroreport 1996;7:156976.

[28] LaBar KS, Phelps EA. Arousal - mediated memory consolidation. Psychological Science 1998;9:490-3.

[29] Lane RD, Reiman EM, Ahern GL, Schwartz GE, Davidson RJ. Neuroanatomical correlates of happiness, sadness, and disgust. American Journal of Psychiatry 1997;154:926-33.

[30] LeDoux JE. Emotion circuits in the brain. Annual Review of Neuroscience 2000;23:155-84.

[31] Maddock RJ. The retrosplenial cortex and emotion: new insights from functional neuroimaging of the human brain. Trends in Neurosciences 1999;22:310-6.

[32] Maddock RJ, Buonocore MH. Activation of left posterior cingulate gyrus by the auditory presentation of threat-related words: an fMRI study. Psychiatry Research 1997;75:1-14.

[33] Maratos EJ, Rugg MD. Electrophysiological correlates of the retrieval of emotional and non-emotional context. Journal of Cognitive Neuroscience, in press.

[34] Markowitsch HJ. The Anatomical basis of memory. In: Gazzaniga MS, editor. The New Cognitive Neurosciences. Cambridge, MA: MIT Press, 2000:781-96.

[35] Mesulam MM. Patterns in behavioural neuroanatomy: association areas, the limbic system, and hemispheric specialisation. In: Mesulam MM, editor. Principles of Behavioural Neurology. Philadelphia: F.A. Davis Co, 1985:1-70.

[36] Northoff G, Richter A, Gessner M, Schlagenhauf F, Fell J, Baumgart F, et al. Functional dissociation between medial and lateral prefrontal cortical spatiotemporal activation in negative and positive emotions: a combined fMRI/MEG study. Cerebral Cortex 2000;10:93-107.

[37] Nyberg L, McIntosh AR, Houle S, Nilsson LG, Tulving E. Activation of medial temporal structures during episodic memory retrieval. Nature 1996;380:715-7.

[38] Pardo JV, Pardo PJ, Raichle ME. Neural correlates of self-induced dysphoria. American Journal of Psychiatry 1993;150:7139. 
[39] Phelps EA, LaBar KS, Spencer DD. Memory for emotional words following unilateral temporal lobectomy. Brain and Cognition 1997;35:85-109.

[40] Phelps EA, LaBar KS, Anderson AK, Connor KJ, Fulbright RK, Spencer DD. Specifying the contributions of the human amygdala to emotional memory: a case study. Neurocase 1998;4:527-40.

[41] Phillips ML, Bullmore ET, Howard R, Woodruff PW, Wright IC, Williams SC, et al. Investigation of facial recognition memory and happy and sad facial expression perception: an fMRI study. Psychiatry Research 1998;83:127-38.

[42] Phillips ML, Young AW, Scott SK, Calder AJ, Andrew C, Giampietro V, et al. Neural responses to facial and vocal expressions of fear and disgust. Proceedings of the Royal Society of London B Biological Science 1998;265:1809-17.

[43] Reiman EM, Lane RD, Ahern GL, Schwartz GE, Davidson RJ, Friston KJ, et al. Neuroanatomical correlates of externally and internally generated human emotion. American Journal of Psychiatry 1997;154:918-25.

[44] Rogers RD, Owen AM, Middleton HC, Williams EJ, Pickard JD, Sahakian BJ, et al. Choosing between small, likely rewards and large, unlikely rewards activates inferior and orbital prefrontal cortex. Journal of Neuroscience 1999;19:9029-38.

[45] Rolls ET. The orbitofrontal cortex and reward. Cerebral Cortex 2000;10:284-94.

[46] Rolls ET, Hornak J, Wade D, McGrath J. Emotion-related learning in patients with social and emotional changes associated with frontal lobe damage. Journal of Neurology, Neurosurgery and Psychiatry 1994;57:1518-24.

[47] Rugg MD, Henson RN. Episodic memory retrieval: an (eventrelated) functional neuroimaging perspective. In: Parker AE, Wilding EL, Bussey TJ, (Eds.), The Cognitive Neuroscience of Memory Encoding and Retrieval. Psychology Press, in press.

[48] Rugg MD, Fletcher PC, Frith CD, Frackowiak RS, Dolan RJ. Differential activation of the prefrontal cortex in successful and unsuccessful memory retrieval. Brain 1996;119:2073-83.

[49] Rugg MD, Fletcher PC, Frith CD, Frackowiak RS, Dolan RJ.
Brain regions supporting intentional and incidental memory: a PET study. Neuroreport 1997;8:1283-7.

[50] Schacter DL, Alpert NM, Savage CR, Rauch SL, Albert MS. Conscious recollection and the human hippocampal formation: evidence from positron emission tomography. Proceedings of the National Academy of Science USA 1996;93:321-5.

[51] Schacter DL, Buckner RL, Koutstaal W, Dale AM, Rosen BR. Late onset of anterior prefrontal activity during true and false recognition: an event-related fMRI study. Neuroimage 1997;6:259-69.

[52] Shallice T, Fletcher P, Frith CD, Grasby P, Frackowiak RS, Dolan RJ. Brain regions associated with acquisition and retrieval of verbal episodic memory. Nature 1994;368:633-5.

[53] Siegle G. The Balanced Affective Word Project. Web Site http;// www.sci.sdsu.edu/CAL/wordlist/words.prn. Internet. 1998. Ref Type: Electronic Citation

[54] Sprengelmeyer R, Rausch M, Eysel UT, Przuntek H. Neural structures associated with recognition of facial expressions of basic emotions. Proceedings of the Royal Society of London B Biological Science 1998;265:1927-31.

[55] Squire LR, Knowlton BJ. The Medial Temporal Lobe, the Hippocampus and the Memory Systems of the Brain. In: Gazzaniga MS, editor. The New Cognitive Neurosciences. Cambridge, MA: MIT Press, 2000:765-80.

[56] Stark CE, Squire LR. fMRI activity in the medial temporal lobe during recognition memory as a function of study-test interval. Hippocampus 2000;10:329-37.

[57] Talairach J, Tournoux P. Co-Planar Stereotaxic Atlas of The Human Brain. Stuttgart: Thieme, 1988.

[58] Taylor SF, Liberzon I, Fig LM, Decker LR, Minoshima S, Koeppe RA. The effect of emotional content on visual recognition memory: a PET activation study. Neuroimage 1998;8:18897.

[59] Weiskrantz L. Behavioural changes associated with ablation of the amygdaloid complex in monkeys. Journal of Comparative Physiology and Psychology 1956;49:381-91. 